\title{
A virtualidade nas estratégias de inovação aberta: proposta de articulação conceitual
}

\author{
Claudio Pitassi \\ Universidade Estácio de Sá
}

O aprofundamento do uso da ciência em produtos e processos e a distribuição da produção de conhecimento colocaram em xeque a organização chandleriana, favorecendo modelos organizacionais em redes de colaboração. Reconhece-se que as TICs converteram-se em poderosas alavancas para as organizações e estruturas virtuais. Recentemente, ganhou destaque na literatura voltada aos gestores de grandes empresas com tradição em P\&D o modelo de inovação aberta (OI). Também nas estratégias de OI a virtualidade engendrada pelas TICs tem desempenhado um papel determinante, transformando-se em elemento-chave do próprio processo de inovação. Mas a análise do uso da OI - e do papel da virtualidade neste modelo - é praticamente inexistente no caso das firmas brasileiras. Este artigo estende o estudo do uso de TICs em arranjos colaborativos. Seu objetivo é articular as bases conceituais para estudo do papel da virtualidade nas estratégias de inovação aberta pertinentes aos diferentes tipos de redes estratégicas virtuais em que a firma brasileira está inserida. Como resultado principal, o artigo oferece uma proposta de articulação conceitual e descreve seus argumentos centrais.

Palavras-chave: inovação tecnológica; redes; inovação aberta; TICs; virtualidade.

The virtuality in open innovation strategies: proposal of conceptual articulation

The intense use of science in products and processes and the highly distributed character of knowledge production put the chandlerian type of firm in a check position, favoring networks and collaborations arrangements. It is largely assumed that information and communication technologies (ICTs) acted as powerful tools to leverage virtual type of organizations and structures. Recently, the open innovation (OI) model gained great attention on the literature targeted to management. Again, in the OI strategies, the ICTs are having a key role, not only as an enabler of information and knowledge exchange, but also as a critical tool to insert virtuality in the innovation process itself. But the scientific studies about the use of OI strategies, and about the role of virtuality in these models, are almost inexistent for the case of Brazilian firms. This paper extends the study of the use of ICTs in collaboration arrangements. This article's objective is to articulate the conceptual base for the study of virtuality in OI strategies considering the different types of virtual strategic networks in which the Brazilian firms

Artigo recebido em dez. 2010 e aceito em maio 2011. 
are embedded. As it main result, this article offers a proposal of conceptual articulation, describing its central arguments.

KEY WORDs: technology; innovation technology; networks; open innovation; ICTs.

\section{Introdução}

Já há um amplo reconhecimento entre pesquisadores que se apoiaram em diferentes perspectivas para analisar a realidade das economias avançadas de que o pleno acesso a ativos estratégicos, principalmente ao conhecimento, não pode mais ser alcançado pela ação da firma isolada (Cohen e Levinthal, 1990; Coombs e Metcalfe, 2000; Robertson e Verona, 2006). Assim, cresce a defesa da necessidade de se alterarem as estratégias competitivas feitas ao nível da firma isolada para o nível da rede de relacionamentos com parceiros externos (Dyer e Singh, 1998; Gulati, 1998). Segundo Castells (1999), a intensificação da penetração das tecnologias da informação e comunicação (TICs) no ambiente de negócios e na sociedade contribuiu fortemente para que as redes se tornassem uma dimensão analítica preferencial. Especificamente em relação à gestão de $\mathrm{P} \& \mathrm{D}$, argumenta-se que, no paradigma tecnoprodutivo atual, no qual as capacitações e os conhecimentos encontram-se distribuídos, o modelo em rede, em parte habilitado pelas TICs, também se tornou o mais efetivo (Rothwell, 1994).

Recentemente, a defesa de um modelo supostamente "disruptivo" de gestão da inovação, chamado de inovação aberta (open innovation - OI), obteve grande repercussão junto ao management das grandes firmas de economias avançadas com tradição em P\&D (Chesbrough, 2003). A exemplo do que se observou nos casos de organizações virtuais (Venkatraman e Henderson, 1998), estudos empíricos trazem evidências da contribuição das TICs para a mudança da gestão da P\&D destas firmas em direção aos modelos de OI, particularmente no que diz respeito ao uso das TICs como ferramenta-chave para a virtualização do processo inovativo (Dogson et al., 2006; Hacievliyagil, 2007; Von Hippel, 2002). Mas a análise do uso da OI — e do papel da virtualidade neste modelo - é praticamente inexistente no caso das firmas brasileiras.

Este ensaio teórico posiciona-se dentro das tradições que estudaram o uso das TICs para o acesso aos conhecimentos distribuídos nas redes de colaboração em que a firma individual está inserida. Neste artigo, a discussão se concentra na inovação aberta, mas interpretando-a na perspectiva relacional que emerge na economia do conhecimento (EC). O objetivo deste artigo é articular as bases conceituais para estudo do papel das virtualidades nas estratégias de inovação aberta pertinentes aos diferentes tipos de redes estratégicas virtuais em que a firma brasileira está inserida.

Este artigo está organizado da seguinte forma: na seção seguinte apresenta-se o referencial teórico que servirá de base para a articulação conceitual. Na seção três, apresentamse as questões de pesquisa e o arcabouço de trabalho articulado para respondê-las. Por fim, fazem-se as considerações conclusivas, destacando a contribuição do artigo para a teoria e a prática gerencial. 


\section{Referencial teórico}

A relação simbiótica entre a evolução das sociedades humanas, da tecnologia e da cultura em seus mais variados níveis tem sido matéria bastante estudada. Pesquisas vêm demonstrando que a evolução das TICS tem acarretado profundas transformações nas sociedades humanas, sistemas econômicos e modelo de negócio (Capra, 2002; Castells, 1999; Lévy, 1999; Pitassi, 2004). Entre as transformações associadas à EC, ganham destaque nesta pesquisa a importância crescente da inovação aberta e o prevalecimento de estruturas e arranjos virtuais para a busca de ativos estratégicos complementares e para a troca de conhecimentos. Nos próximos parágrafos serão analisados os temas que contribuem para o entrelaçamento destas questões.

\subsection{TICs}

A tecnologia da informação (TI) é uma tecnologia centrada no computador, que permite a coleta, o armazenamento, o processamento e a comunicação da informação. É composta de hardware (por exemplo, computadores, terminais, roteadores, firewalls e smartphones) e software (por exemplo, sistemas operacionais, aplicativos e websites (Turban, Rainer e Potter, 2007). Sistemas de informação (SI), por sua vez, são uma combinação de processos, informação, pessoas e TI organizada para atingir objetivos de negócios. Na perspectiva do campo de estudo das TICs, informação é tudo aquilo que pode ser digitalizado e comunicado na rede, envolvendo dados, voz e imagem (Shapiro e Varian, 1999). A revolução da microeletrônica no final da década de 1940 e a difusão das TICs ao longo da segunda metade do século passado, particularmente da Internet e da WWW, propiciaram o surgimento de uma economia digital, na qual o potencial de conexão entre objetos, pessoas, empresas e países é teoricamente ilimitado (Castells, 1999). Por estas razões, as TICs são vistas como um elemento central, que devem merecer uma atenção cada vez maior dos administradores contemporâneos (Albertin, 2001).

Desde a chegada do mainframe ao ambiente de negócios, as TICs vêm ampliando seu espaço de aplicação nas organizações. Hoje, reconhece-se que elas habilitam o redesenho de processos de negócio (Keen, 1991), o tratamento de informações cada vez mais complexas e uma ampla conectividade entre agentes econômicos (Zuboff, 1988). Também se argumenta que o desenvolvimento da indústria de TI influenciou na proliferação de arquiteturas modulares e abertas em outras indústrias (Sanchez, 1999) e no desenvolvimento das demais tecnologias de propósito geral, tal como a biotecnologia (Castells, 1999). No início desta década, o impacto das TICs vem se aprofundando ainda mais, convertendo-se em um mecanismo de virtualização das relações sociais (Lévy, 1999), de modelos econômicos (Venkatramam e Henderson, 1998) e do próprio processo inovativo (Dogson et al., 2006), ampliando seu potencial de virtualização das áreas de P\&D (Pitassi; 2011).

$\mathrm{Na}$ perspectiva deste artigo, interessa avaliar como as TICs influenciam na maneira como o conhecimento é criado e compartilhado nas redes de colaboração e como elas podem 
influenciar na virtualização da P\&D. A tabela 1 resume os tipos de TICs aplicados ao processo inovativo nas estratégias de colaboração em P\&D encontrados na literatura investigada.

Tabela 1

Uso das TICs nas estratégias de colaboração em P\&D

\begin{tabular}{|c|c|c|}
\hline Tipos de TICs & Objetivo & Referência \\
\hline $\begin{array}{l}\text { Comunidades open source para } \\
\text { colaboração virtual }\end{array}$ & $\begin{array}{l}\text { Participação em comunidade nas quais todos } \\
\text { podem oferecer e solicitar ideias, mas não há } \\
\text { controle sobre a inovação }\end{array}$ & $\begin{array}{l}\text { Chesbrough e Appleyard (2007); Pisano e } \\
\text { Verganti (2008). }\end{array}$ \\
\hline $\begin{array}{l}\text { Sistemas de Gestão do conheci- } \\
\text { mento na cadeia produtiva }\end{array}$ & $\begin{array}{l}\text { Sistemas de informação utilizados na identifi- } \\
\text { cação, criação, apresentação e disseminação } \\
\text { de conhecimento }\end{array}$ & $\begin{array}{l}\text { Alavi e Leidner (2001); Borghoff e Pareschi } \\
\text { (1998). }\end{array}$ \\
\hline Data minning e busca & $\begin{array}{l}\text { Uso de técnicas de mineração de dados na } \\
\text { manipulação de documentos e compartilha- } \\
\text { mento de informação }\end{array}$ & $\begin{array}{l}\text { Dogson e colaboradores (2006); Sakkab } \\
(2002)\end{array}$ \\
\hline Simulação e modelagem & $\begin{array}{l}\text { Aplicação de técnicas de simulação de cenários } \\
\text { e otimização de processos }\end{array}$ & $\begin{array}{l}\text { Brown (2003); Dogson e colaboradores } \\
\text { (2006) }\end{array}$ \\
\hline Prototipação e visualização virtual & $\begin{array}{l}\text { Criação no design colaborativo de protótipos } \\
\text { virtuais de embalagens, produtos e processos } \\
\text { produtivos }\end{array}$ & $\begin{array}{l}\text { Dogson e colaboradores (2006); Thomke, } \\
(2003)\end{array}$ \\
\hline Toolkits para clientes & $\begin{array}{l}\text { Ferramentas amigáveis de } \mathrm{TI} \text { oferecidas aos } \\
\text { clientes para habilitá-los a participar no desen- } \\
\text { volvimento de seus produtos }\end{array}$ & Nambisan (2002); Von Hippel (2002) \\
\hline $\begin{array}{l}\text { Portais próprios com funcionalida- } \\
\text { des para apoiar a inovação }\end{array}$ & $\begin{array}{l}\text { Uso da internet como meio regular de cone- } \\
\text { xão com parceiros envolvidos no processo } \\
\text { inovativo }\end{array}$ & Sakkab (2002); Pisano e Verganti (2008) \\
\hline Virtual Knowledge Brokers & $\begin{array}{l}\text { Uso de infomediários que alavancam a capa- } \\
\text { cidade da internet de acessar o conhecimento } \\
\text { disponível no mercado }\end{array}$ & $\begin{array}{l}\text { Hacievliyagil (2007); Verona e colabora- } \\
\text { dores (2006) }\end{array}$ \\
\hline
\end{tabular}

Fonte: elaboração própria.

\subsection{Inovação tecnológica}

A importância da inovação tecnológica para o crescimento das firmas e para o desenvolvimento econômico dos países está solidamente apoiada por um conjunto de obras seminais produzidas na literatura de economia industrial (Bell e Pavitt, 1995; Dosi, 1982; Freeman e Soete, 1997; Nelson e Winter, 1982; Pavitt, 1984, 1990; Penrose, 1959; Rosenberg, 1982; Shumpeter, 1934; Teece et al., 1997). A história também nos revela que a fronteira tecnológica está sempre em movimento (Landes, 1969), abrindo oportunidades, nos momentos de mudanças de paradigma tecnológico, para o fechamento do gap tecnológico (catch-up) das firmas de países emergentes (Lee e Lim, 2001). 
Para compreender a dinâmica da inovação tecnológica é preciso, em primeiro lugar, enfatizar que a inovação não é igual à invenção, e que a tecnologia não é igual à ciência (Freeman e Soete, 1997), muito embora estes fenômenos estejam fundamentalmente conectados (Dosi, 1982). Como afirma Utterback (1971:77), a inovação nasce de uma invenção que foi capaz de se materializar e que, portanto, altera substancialmente um processo ou alcança o mercado com a introdução de um novo produto ou serviço. Também não significa que inovar é fazer o que ninguém fez em lugar nenhum, pois se uma firma imita um concorrente inovador, isto também é inovação do ponto de vista da firma que copia (Teece, 1986).

Dentro do contexto organizacional, é adequado diferenciar a inovação, no sentido lato, da inovação tecnológica, particularmente daquela baseada em ciência. Para tal, precisamos definir com mais profundidade do que é usualmente feito na literatura não científica o conceito de tecnologia. A tecnologia tem uma natureza tácita e difusa que envolve múltiplos conhecimentos e processos de aprendizado individual e coletivo (Cohen e Levinthal, 1990), bem como aspectos organizacionais e institucionais que são específicos ao contexto nos quais eles se desenvolvem (Dosi, 1982; Rosenberg, 1982; Pavitt, 1990). Por conseguinte, fazer uma inovação tecnológica significa avançar a base de conhecimentos que estão embebidos no tecido organizacional da firma e que serão aplicados a produtos e processos diferenciados (Teece et al., 1997). No paradigma tecnoprodutivo associado à EC, mudar as técnicas aplicadas a processos não depende apenas das habilidades das mãos e dos olhos, do "aprender fazendo", mas também do domínio de princípios científicos (Freeman e Soete, 1997).

Quando ocorrem, as inovações tecnológicas podem ser mais ou menos intensas quanto a seus efeitos disruptivos nas trajetórias tecnológicas em curso. Em função destes impactos, inovações tecnológicas podem ser divididas em autônoma versus sistêmica (Chesbrough e Teece, 1996), ou em incremental versus radical (Sivadas e Dwyer, 2000). As inovações sistêmicas ou radicais desencadeiam novas trajetórias e tecnologias que precisam de um longo e incerto processo de difusão até se transformarem em produtos e serviços comercializáveis (Freeman e Soete, 1997). Já as inovações autônomas ou incrementais são aquelas que podem ser realizadas independentemente de outras inovações na cadeia produtiva.

A opção, no contexto da firma, por enfrentar ou não as incertezas da inovação radical nos remete à questão do posicionamento tecnológico pretendido. O posicionamento tecnológico reflete a intensidade dos esforços de inovação da empresa e envolve a demarcação de como ela irá conduzir o processo de obtenção das tecnologias-chave para sua estratégia empresarial. Bell e Pavitt (1995), adaptando a tipologia proposta originalmente por Pavitt (1984), argumentam que os setores e empresas têm padrões de inovação específicos, que influenciam as ações inovativas das firmas aí presentes. Segundo Pisano (2006), as mudanças na natureza da propriedade intelectual, associadas ao paradigma tecnoprodutivo atual, trouxeram novos desafios para as firmas no que diz respeito a sua capacidade de influenciar os regimes de apropriação dos resultados da inovação tecnológica, favorecendo, em alguns casos, a adoção de modelos de código aberto. 


\subsection{A visão sistêmica ou relacional da inovação tecnológica}

A necessidade de aprofundar o estudo da relação entre o progresso técnico e o desenvolvimento econômico dos países fez com que economistas associados à corrente evolucionária neoschumpeteriana cunhassem o conceito de SNI (Lundvall, 1992), que ainda hoje exerce forte influência nas políticas de C\&T ao redor do mundo, servindo de base teórica para o Manual de Oslo da OECD. O caráter sistêmico deixa claro que o progresso técnico constituía-se em um processo social, histórico e cumulativo de trajetórias tecnológicas, influenciado pela interação entre a organização e as instituições que a circundam (Dosi, 1982). Dado seu marco teórico, a visão sistêmica foi o referencial dominante nos estudos de sistemas tecnológicos e industriais nos níveis nacional, setorial, regional e local. No caso dos estudos de clusters ou distritos industriais, a análise sistêmica da inovação enfatiza os benefícios das externalidades positivas geradas pela troca de conhecimentos tácitos, específicos e localizados, possibilitados pelo aprendizado gerado pela proximidade geográfica entre os indivíduos e as organizações (Audretsch e Feldman, 1996). Entretanto, com o aprofundamento do uso das TICs nas empresas e na sociedade, argumenta-se que os efeitos de spillovers, ressaltados nos estudos dos clusters industriais, não dependeriam tanto da proximidade física, dada a independência de tempo e espaço que elas (as TICs) proporcionam (Dantas e Bell, 2009).

\subsection{Gestão do conhecimento}

A tecnologia é um tipo de conhecimento voltado à engenharia, ou seja, à solução de problemas práticos de empresas e da sociedade (Figueiredo, 2009). De acordo com Nambisam (2002), a cogeração de conhecimento habilitado pelas TICs deve ser analisada partindo-se da perspectiva sociológica de criação de conhecimento e valor (Nonaka, 1994), o que demanda o entendimento do que significam conhecimento e aprendizado organizacional, os quais, desde meados da década de 1980, desempenham papel central em arcabouços analíticos de diferentes tradições teóricas (Grant, 1996; Kogut e Zander, 1992; Levitt e March, 1988; Nonaka, 1994), particularmente no que tange aos conceitos de capacidade absortiva (Cohen e Levinthal, 1990), capacidades tecnológicas (Bell e Pavitt, 1995) e capacidade dinâmica (Teece et al., 1997), todos fundamentais para o estudo da inovação.

Aprendizado organizacional é aqui definido como o processo por meio do qual o conhecimento é criado, distribuído e comunicado na organização (Kim, 1998). No que diz respeito à aprendizagem tecnológica, Figueiredo (2009) destaca ser ela o processo que permite a uma empresa acumular capacidade tecnológica ao longo do tempo. Na vertente sociológica mencionada acima, o processo de criação de conhecimento é um fenômeno social complexo e multifacetado. Para Nonaka (1994), a aprendizagem organizacional é um processo dinâmico, chamado de espiral de criação de conhecimento, o qual apresenta duas dimensões: i) epistemológica, que define duas categorias de conhecimento, o tácito e o explícito (Polanyi, 1966); ii) ontológica, que define o nível de interação social na criação, indo do conhecimento individual ao interorganizacional. 
Nas organizações, o conhecimento tático (know-how) é aquele que está acumulado nas mentes dos especialistas e nas rotinas e heurísticas organizacionais; já o conhecimento explícito (know about) é aquele que está codificado em padrões e procedimentos operacionais internos ou que pode ser encontrado em livros técnicos e manuais disponíveis no mercado (Grant, 1996). No processo de aprendizado, os indivíduos desempenham o papel principal, mas a capacidade da firma de integrar os conhecimentos dos especialistas e transformá-los em produtos e processos diferenciados é o que confere ao aprendizado em nível organizacional seu caráter sinérgico (Grant, 1996).

Neste artigo, diremos que o conhecimento permite que um fenômeno ou uma nova situação façam sentido para uma pessoa, conferindo-lhe uma habilidade e uma capacidade de agir e de conscientemente criar significados e encontrar soluções aceitáveis (Nonaka e Takeuchi, 1995). De acordo com Dutrénit (2001), observa-se em muitas organizações um conhecimento que permanece no estágio tácito, muito embora pudesse vir a ser codificado, facilitando o funcionamento da espiral do conhecimento. Ainda segundo a autora, um conhecimento tácito pode, com o passar do tempo e com maior domínio científico nele envolvido, ser codificado, facilitando sua disseminação. O papel das TICs na gestão do conhecimento, para o funcionamento especificamente da espiral do conhecimento, tem merecido destaque na literatura de TI desde o fim da década de 1990 (Alavi e Leidner, 2001; Borghoff e Pareschi, 1998).

\subsection{A organização pós-chandleriana}

Segundo Chandler (1977), a emergência da grande empresa verticalizada, que dominou praticamente todos os setores econômicos gestados a partir da Segunda Revolução Industrial, foi uma resposta às ineficiências impostas pelas transações de mercado, permitindo que a firma atuasse em escala global, com a gestão eficiente de diversas atividades econômicas e a produção e o fornecimento de diferentes mercadorias e serviços. Na busca pelos benefícios das economias de escala e escopo, estas firmas procuraram, ao longo de três quartos do século passado, aumentar seus volumes de produção e verticalizar para a frente e para trás suas operações, internalizando ativos complementares que protegessem seus investimentos em plantas e equipamentos (Robertson e Verona, 2006). Para aumentar seus ganhos de escala e escopo, a firma chandleriana criou os centros profissionais de $\mathrm{P} \& \mathrm{D}$, cujas inovações buscadas envolviam conhecimentos específicos à sua atividade econômica e inexistentes no mercado (Chesbrough, 2006). Estas estruturas constituíram-se em poderosas barreiras à entrada de competidores (Teece, 1986).

Com a revolução da microeletrônica, a difusão das TICs e as transformações tecnoprodutivas associadas à EC, as vantagens da grande empresa verticalizada perdem relevância diante dos benefícios do acesso a conhecimentos distribuídos entre um número cada vez maior de empresas especialistas. Segundo Coombs e Metcalfe (2000), três fenômenos explicam a crescente especialização do conhecimento: i) com o aprofundamento do uso de ciência nas empresas, os produtos e processos passam a embutir um amplo espectro de tecnologias e conhecimentos constituintes, o que torna o domínio interno de todos eles inviável ou mesmo impossível; ii) os novos produtos e processos desempenham um papel crescente de compo- 
nentes de um sistema complexo e, cada vez mais, de arquiteturas modulares (Sanchez, 1999); iii) os desbalanceamentos contínuos nas capacitações de cada firma e a dinâmica da divisão do trabalho na produção de conhecimento fazem com que a delimitação da fronteira da firma em relação ao ambiente torne-se cada vez menos clara, forçando uma revisão constante nos conhecimentos que a firma precisa dominar internamente.

\subsection{Redes de colaboração com parceiros externos}

O entendimento de que a organização mantém uma relação sistêmica com o ambiente no qual atua consolida-se com a ecologia organizacional, que se desenvolve a partir da segunda metade dos anos de 1970 (Hannan e Freeman, 1977). A imagem da organização incrustada (embedded) na teia de relações sociais que estabelece com outras organizações (Uzzi, 1997) é a base para as teorias de redes (networks) que ganham corpo na literatura de estratégia (Gulati, 1998), de marketing (Achrol e Kotler, 1999) e de supply chain (Chandrashekar e Schary, 1999) no final da década de 1990. De acordo com Gulati e colaboradores (2000:203), as redes estratégicas compreendem um conjunto de relacionamentos da empresa, tanto horizontais como verticais, com outras organizações - fornecedores, clientes, concorrentes e outras entidades —, incluindo relações entre empresas de diferentes indústrias e países. São compostas de laços interorganizacionais duradouros, de significado estratégico, e incluem alianças estratégicas, joint ventures e parcerias de longo prazo com fornecedores. Podem ser decompostas em vários laços bilaterais envolvendo motivos de caráter colaborativo e competitivo, em que os parceiros possuem interesses particulares e comuns.

As diferentes correntes teóricas citadas acima têm em comum o entendimento de que a escala e o controle interno dos recursos alocados nas estratégias de negócio, percebidos como fontes de vantagem competitiva das organizações verticalmente integradas no período de ouro da economia industrial, não foram capazes de responder às exigências crescentes dos mercados, acelerando o processo de emergência de novos arranjos de negócios em rede (Gulati, 1998; Häcki e Lighton, 2001; Jarrillo, 1998). Na medida em que a convergência e a retroalimentação entre as exigências organizacionais e as transformações tecnológicas avançaram, a integração em redes transformou-se em uma alternativa viável de concorrência, pois se tornou impossível para a organização individual construir todas as competências de que necessita (Castells, 1999).

Na perspectiva de capacidades distribuídas (distributed capabilities) (Coombs e Metcalfe, 2000; Dantas e Bell, 2009), as redes usam rotinas organizacionais que permitem a cada parceiro complementar os conhecimentos necessários à geração e difusão de novos produtos e processos, usando a especialização proposital e fluxos multidirecionais complementares de transferência de conhecimento especializado entre os parceiros. Os estudos de redes interorganizacionais também foram diretamente aplicados à questão da mudança técnica e sua dinâmica (Malerba, 2002; Orsenigo et al., 2001), incluindo o caráter distribuído e global das fontes, ressaltando nestas análises o papel habilitador da troca de informação e conhecimentos das TICs (Rothwell, 1994). 
A virtualidade (Lévy, 1999) catalisada pela evolução da TI nos modelos de negócio (Castells, 1999) vem habilitando a quebra de estruturas rigidamente coordenadas, típicas de redes tradicionais, permitindo o desenvolvimento de redes com escopo variável e dinâmico (Pitassi e Macedo-Soares, 2003). Autores que estudaram o fenômeno das organizações virtuais não demarcam com clareza as fronteiras entre os modelos convencionais e os modelos de rede habilitados pelas TICs (Castells, 1999; Markus, Manville e Agres, 2000; Venkatraman e Henderson, 1998), o que deixa o leitor confuso sobre possíveis diferenças. Para auxiliar neste entendimento, Pitassi (2004:51-52) definiu que

quando consideramos a infraestrutura de comunicações que são utilizadas na criação e manutenção das redes estratégicas, podemos vislumbrar um espectro em cujas extremidades encontram-se, de um lado, as redes estratégicas tradicionais (RE) e, do outro lado, as redes estratégicas virtuais puras (RV). Entre esses dois formatos limítrofes, existem infinitas possibilidades de combinações na formação das Redes Estratégicas. Chamaremos esses formatos intermediários de REVs.

As comunidades virtuais, tais como os portais de e.procurement ou os portais de inovação, podem ser consideradas exemplos de formas puras (Pitassi e Dias, 2003).

A palavra virtual vem do latim medieval virtuallis, que por sua vez deriva de virtus, que significa força e potência (Lévy, 1999). Logo, estas estruturas poderão ampliar o potencial de alocação e alavancagem de recursos das redes, na medida em que possibilitem a recuperação descentralizada de informações e sua integração simultânea em um sistema flexível e multifacetado de simulação e elaboração de estratégias, as quais não poderiam ser alcançadas a partir de cada firma individual (Castells, 1999). Dada a mudança de patamar nos mecanismos de gestação e implantação de proposições de valor alternativas, e até simultâneas, a virtualização das redes pode ser vista como a emergência de estruturas organizacionais adequadas à competição na era do caos, dentro da lógica de sistemas complexos, nos quais é impossível prever-se o estado futuro em que a firma atuará (Faucheux, 1997; Mowshowitz, 1997; Black e Edwards, 2000).

\subsection{Inovação aberta - Open innovation (OI)}

Recentemente, ganhou corpo na literatura voltada aos gestores o modelo de inovação aberta. De acordo com Chesbrough e Vanhaverbeke (2006:2), a OI caracteriza-se pelo "uso intencional de fluxos de entrada e de saída de conhecimentos com o propósito de respectivamente acelerar a inovação interna e expandir os mercados para o uso externo de inovação". Embora os riscos e custos da inovação tecnológica sejam maiores em setores de base tecnológica, tais como informática e biotecnologia, pesquisas revelam que a adoção da OI não se restringe a estas indústrias (Chesbrough e Crowther, 2006). A revisão da incipiente literatura científica de OI sugere que, por um lado, a OI estende as discussões das diferentes correntes que reconheceram a relevância estratégica do acesso a conhecimentos externos, mantendo com elas uma forte conexão quanto aos temas estudados (Dahlander e Gann, 2010). Por outro, a OI busca 
avançar na interpretação das transformações que vem sofrendo a organização pós-chandleriana, particularmente no que diz respeito ao caráter distribuído e crescentemente especializado da produção dos conhecimentos (Chesbrough, 2006).

Para Chesbrough (2003), o ambiente de negócios contemporâneo, ao desfavorecer estratégias competitivas que forçassem a firma a fazer tudo sozinha, colocou os modelos verticalizados de gestão de $\mathrm{P} \& \mathrm{D}$, com sua ênfase no segredo industrial, em xeque quanto à sua capacidade de inovar rapidamente e de enfrentar os desafios da competição em escala global. Na perspectiva da OI, contribuíram para a emergência do modelo aberto: i) a mobilidade crescente de engenheiros e especialistas que levam com eles os conhecimentos adquiridos; ii) a qualidade e a orientação para o mercado da pesquisa feita em universidades de ponta ao redor do mundo; iii) a disseminação global do conhecimento; iv) a rivalidade crescente nos mercados globais; v) a emergência dos venture capitalists dispostos a levar à frente ideias inovadoras.

Na perspectiva da OI, os vários casos de empresas sem estruturas internas de P\&D expressivas, mas com reconhecido sucesso inovativo, tais como Cisco e Staples, comprovariam que, na EC, não é mais necessário gerar internamente inovação para uma firma usufruir dela (Chesbrough, 2003). Para Chesbrough (2006), a capacidade de a firma desenvolver modelos de negócios alternativos é de crucial importância para que ela faça melhor uso das tecnologias à sua disposição. Modelos de negócios são descritos pelo autor (Chesbrough, 2006:12) "como um instrumento cognitivo que influencia o foco da análise dos projetos de P\&D dentro da firma".

O caráter distribuído e fragmentado da produção de conhecimento, enfatizado na OI, fez brotar fontes não convencionais que podem ser usadas no processo de inovação, sejam elas vindas de fornecedores de ideias incipientes para aprofundamento na fase de pesquisa aplicada, de conceitos prontos para o desenvolvimento ou mesmo da oferta à firma de produtos prontos para entrar no mercado. Os modelos verticalizados e lineares de P\&D refletiram a preocupação crescente das empresas com os "falsos positivos", dada a magnitude das perdas financeiras envolvidas (Chesbrough, 2006).

No sentido da saída do fluxo de conhecimento, a OI enfatiza que a baixa capacidade de as firmas perceberem os "falsos negativos" que brotam na gestão de P\&D pode fragilizar seriamente sua posição competitiva no longo prazo. Entre os mecanismos propostos pelo OI para mitigar este problema, destacam-se: i) o licenciamento para terceiros de ideias, conceitos ou patentes gerados no passado e que estão em estado de espera; ii) a participação em firmas startups ou spin-offs que levem à frente as inovações consideradas distantes das trajetórias tecnológicas atuais, mas que possam gerar opções reais de acesso a novos mercados para a firma no futuro. Dada a dinâmica de entrada e saída de conhecimentos e a inter-relação dos dois fluxos, a OI propõe uma gestão proativa — no limite, de mercado — da propriedade intelectual (Chesbrough, 2006). Pesquisas evidenciam que as TICs têm colaborado para a implantação dos modelos de OI e para a virtualização do processo de inovação aberta (Dogson et al., 2006).

Apoiando-se na proposição de Chesbrough (2006:15-16) sobre aspectos cujo uso intensivo na gestão da P\&D na empresa permite diferenciar a OI dos demais estudos que ressaltaram a importância das fontes externas de conhecimento, a tabela 2 apresenta a lista de atributos da OI que serão considerados neste estudo: 
Tabela 2

\section{Atributos distintivos da OI}

\begin{tabular}{|c|c|}
\hline Atributos & Fator de diferenciação da OI \\
\hline $\begin{array}{l}\text { Percepção do cenário de geração do } \\
\text { conhecimento }\end{array}$ & $\begin{array}{l}\text { Percepção do cenário de geração externa de conhecimentos como relevante, de } \\
\text { qualidade, disperso e acessível. Firma rechaça a síndrome NIH (not invented here) e } \\
\text { utiliza inteligência competitiva tecnológica. }\end{array}$ \\
\hline $\begin{array}{l}\text { Importância relativa das fontes } \\
\text { de conhecimento na cultura } \\
\text { organizacional }\end{array}$ & $\begin{array}{l}\text { Peso equivalente (não acessório) das fontes externas de conhecimento vis-à-vis as } \\
\text { fontes internas de conhecimentos. A inovação pode entrar e sair em qualquer fase da } \\
\text { P\&D. }\end{array}$ \\
\hline $\begin{array}{l}\text { Papel central do Modelo de } \\
\text { Negócios na gestão da P\&D }\end{array}$ & $\begin{array}{l}\text { O modelo de negócios adotado pela firma influencia diretamente na forma como a } \\
\text { empresa opta por transformar tecnologia em bens e serviços. O importante não é a } \\
\text { tecnologia, mais sim a proposição de valor ao cliente. }\end{array}$ \\
\hline Prontidão em aquisições & $\begin{array}{l}\text { Agilidade para efetivar e concluir aquisições de empresas inovadoras de base } \\
\text { tecnológica identificadas como oportunidades. }\end{array}$ \\
\hline $\begin{array}{l}\text { Uso de parcerias de } \\
\text { codesenvolvimento }\end{array}$ & $\begin{array}{l}\text { Capacidade de conduzir - ou participar de - projetos com parceiros externos, } \\
\text { garantindo a qualidade e a memória técnica dos desenvolvimentos efetuados. }\end{array}$ \\
\hline $\begin{array}{l}\text { Mitigação das falhas nos projetos } \\
\text { de P\&D }\end{array}$ & $\begin{array}{l}\text { Tomando por base seu modelo de negócio, as firmas usam processos para mitigar } \\
\text { tanto os falsos positivos quanto os falsos negativos. }\end{array}$ \\
\hline $\begin{array}{l}\text { Importância dos fluxos de saída de } \\
\text { conhecimentos }\end{array}$ & $\begin{array}{l}\text { Uso proativo dos conhecimentos não diretamente relacionados às trajetórias } \\
\text { tecnológicas principais, traduzido na capacidade de geração de spin-offs ou de } \\
\text { financiar empresas incubadas. }\end{array}$ \\
\hline $\begin{array}{l}\text { Modelo de gestão da propriedade } \\
\text { intelectual }\end{array}$ & $\begin{array}{l}\text { A gestão proativa e integrada da propriedade intelectual, tanto no sentido de entrada } \\
\text { quanto no de saída, torna-se um elemento-chave na gestão da } \mathrm{P} \& \mathrm{D} \text {, permitindo à } \\
\text { empresa atuar no mercado de tecnologia. }\end{array}$ \\
\hline $\begin{array}{l}\text { Importância dos intermediários na } \\
\text { cadeia de inovação }\end{array}$ & $\begin{array}{l}\text { Os novos intermediários assumem um papel direto na cadeia de inovação da firma, } \\
\text { realizando atividades reconhecidamente relevantes da P\&D, antes mantidas internamente. }\end{array}$ \\
\hline Intensidade no uso de TICs & $\begin{array}{l}\text { Existência e domínio sobre sistemas de TICs capazes de gerenciar todo o fluxo de } \\
\text { ideias na empresa e na articulação externa em redes. Há uma preocupação crescente } \\
\text { com a codificação do conhecimento. }\end{array}$ \\
\hline $\begin{array}{l}\text { Métricas de avaliação de desempenho } \\
\text { da P\&D }\end{array}$ & $\begin{array}{l}\text { Ao lado das tradicionais, as empresas adotam métricas voltadas aos esforços conjuntos } \\
\text { de P\&D na cadeia de suprimentos e no uso comercial de propriedade intelectual gerada } \\
\text { pela firma. }\end{array}$ \\
\hline
\end{tabular}

Fonte: Apoiado em Chesbrough (2006).

O foco deste estudo é a contribuição das TICs nas estratégias de OI. Mesmo reconhecendo os esforços recentes no sentido de dar maior consistência teórica para a pesquisa empírica (Hughes e Wareham, 2010), regra geral, a literatura de OI ainda se encontra em um nível descritivo e genérico, com poucos constructos para a operacionalização da pesquisa. Uma parte da literatura examinada deixa claro que não há um modelo de inovação aberta que sirva a todas as estratégias de inovação (Pisano e Verganti, 2008). Cada situação dependerá de uma complexa combinação de fatores, tais como: ramo de atuação, estratégia empresarial, modelo 
de negócio, cultura organizacional, posicionamento tecnológico, maturidade das trajetórias tecnológicas etc. Por exemplo, segundo Pisano e Verganti (2008), o grau de modularização do conhecimento terá influência na natureza de dinâmica do conhecimento trocado, podendo, para os casos nos quais as interfaces entre os conhecimentos trocados já estejam definidas, assumir a forma extrema dos movimentos de código aberto.

As evidências empíricas de rendimentos decrescentes no uso desmedido de fontes externas de conhecimento (Laursen e Salter, 2006) recomendam um aprofundamento maior da questão, exigindo do pesquisador um olhar crítico (Trott e Hartmann, 2009) sobre o argumento, muito comum na literatura direcionada aos gestores (Huston e Sakkab, 2006), do caráter "disruptivo" da OI na gestão da P\&D da firma. Neste sentido, mesmo com a ênfase dada pelo OI aos fluxos de entrada e saída de conhecimento, um leitura cuidadosa dos escritos de Chesbrough, e dos estudiosos que procuram dar maior consistência analítica ao modelo, sugere a importância de as empresas manterem sua capacidade interna de absorver (Cohen e Levinthal, 1990) os conhecimentos pretendidos. Como enfatizam Cohen e Levinthal em seu estudo seminal, esta capacidade depende da capacidade cognitiva interna de fazer sentido e apropriar-se do conhecimento externo, dada pelo acúmulo interno de conhecimentos e pelos esforços internos de aprendizado.

Visando contribuir para os esforços no sentido de dar maior consistência teórica aos condicionantes do funcionamento da OI e dos mecanismos e processos de aprendizado aí utilizados, esta pesquisa examinará o uso do modelo no contexto das redes estratégicas virtuais nas quais a firma participa.

\section{Arcabouço analítico}

Construída a partir dos estudos e arcabouços teóricos apresentados acima, esta pesquisa examina a relação entre a gestão de $\mathrm{P} \& \mathrm{D}$, a virtualidade e o uso dos atributos da OI nas REVs nas quais estas firmas participam. Para atingir este objetivo, são formuladas três questões de pesquisa:

1. Como as firmas brasileiras estão usando os atributos da inovação aberta nos diferentes tipos de REVs nos quais elas participam?

2. Que tipos de TICs são utilizados e qual o papel delas para a consolidação dos objetivos da inovação aberta pertinentes aos diferentes tipos de REVs?

3. Mais especificamente, de que maneira a virtualidade está afetando a estrutura das áreas de $\mathrm{P} \& \mathrm{D}$ e os mecanismos e processos de aprendizado utilizados nas estratégias de open innovation dentro de cada tipo de REVs?

A articulação dos construtos subjacentes a estas questões de pesquisa contribui para a constituição do arcabouço de trabalho a ser aplicado nesta pesquisa, cujo primeiro esforço de aproximação pode ser visto na figura 1: 
Figura 1

Arcabouço para avaliação do uso de TI nas estratégias de inovação aberta

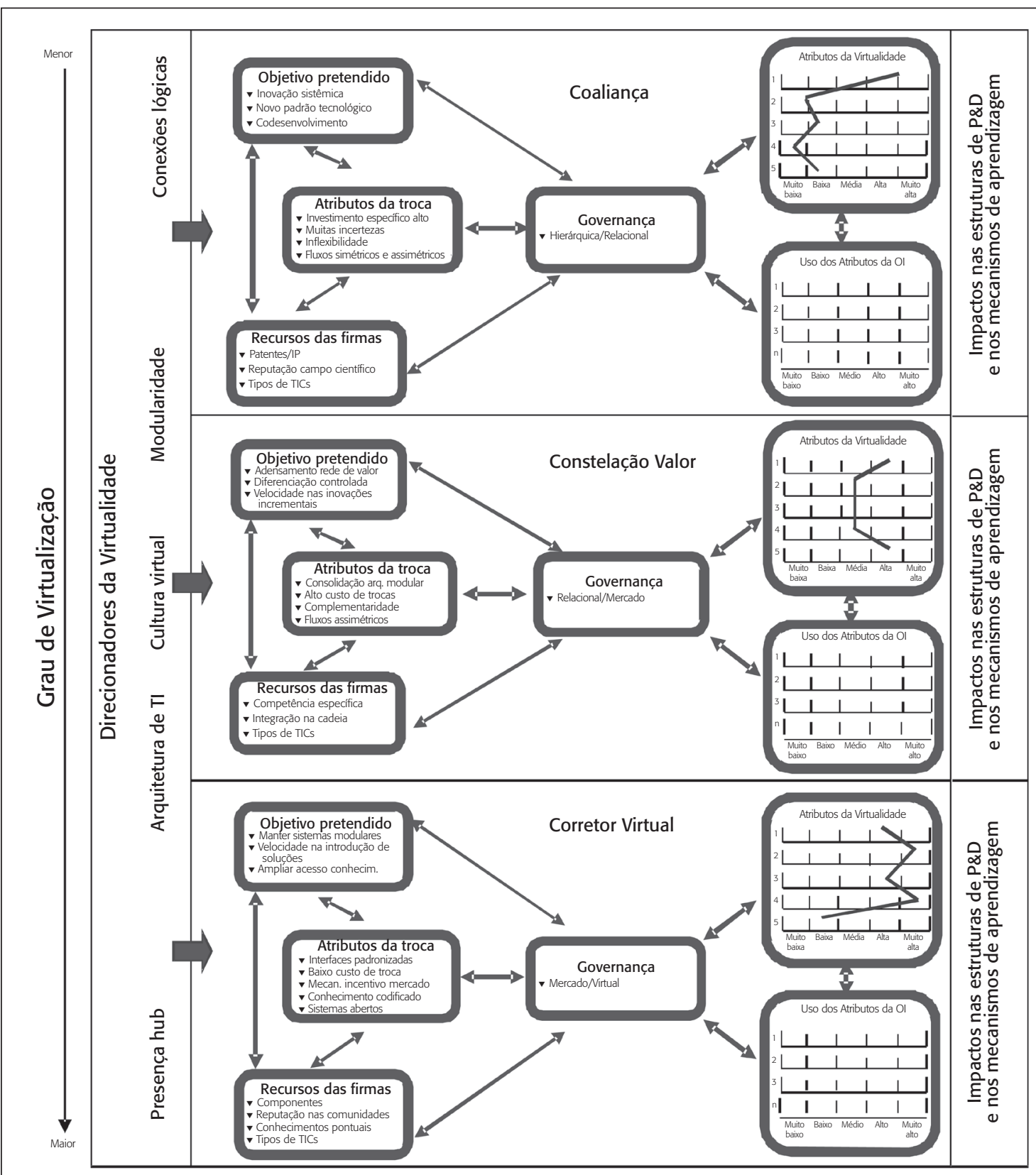

Fonte: Adaptado de Pitassi (2004) e de Ghosh e John (1999). 
O arcabouço proposto argumenta que as estratégias de OI dependem dos objetivos pretendidos, ocasionando impactos distintos nas estruturas de P\&D e nos mecanismos e processos de aprendizagem tecnológica adotados. Cada tipo de REV está associado a um conjunto de atributos trocados na rede, demandando um elenco de recursos de cada firma participante, com destaque para os tipos de TICs aportados e compartilhados. A efetividade das trocas é condicionada pelos mecanismos de governança adotados, o que condiciona o caminho na direção de modelos de rede abertos e flats. O caminho na direção de modelos de código aberto depende da ação conjugada dos direcionadores da virtualidade, o que pode ser medido pela intensidade dos atributos da virtualidade, e do uso pelas empresas participantes dos atributos da OI.

Em termos gerais, defende-se que a governança de mercado adequa-se ao modelo horizontal, de arquitetura modular e cujas inovações tecnológicas pretendidas sejam incrementais. O objetivo da arquitetura modular é estabelecer um padrão de interface, de modo que os componentes possam ser alterados e substituídos sem que seja necessário alterar a arquitetura do produto (Sanchez, 1999). Os modelos verticalizados, por seu turno, seriam mais efetivos em momentos de inovação sistêmica, quando o padrão dominante ainda não está definido (Chesbrough e Teece, 1996). Assim, no caso da reconfiguração da cadeia de inovação no contexto de virtualização crescente, cabe criar um novo paradigma de governança. Uma rápida explicação sobre cada tipo de REV ajudará na compreensão do arcabouço.

Nas coalianças, cada parceiro compromete-se igualmente com a rede, formando uma espécie de consórcio com laços relativamente estáveis, geralmente contratuais e voltados para objetivos específicos, como a colaboração nas fases iniciais dos projetos de P\&D. Nos casos em que há uma empresa central, esta seleciona os parceiros que considera os mais capacitados nos conhecimentos subjacentes à solução pretendida, mantendo rígido controle sobre a evolução da pesquisa e sobre quem captura o valor da inovação. Este modelo é mais adotado nos momentos de inovação radical ou nas fases iniciais da pesquisa, quando a direção correta não está clara. Os maiores desafios são a identificação dos campos de conhecimentos subjacentes, os parceiros mais capacitados e a escolha da direção correta. Nos casos onde há mútua dependência e as múltiplas direções dos conhecimentos trocados desfavorecem tentativas de controle sobre a captura do valor gerado com a inovação, não haveria, a rigor, uma posição central. Este modo é recomendado em ramos cuja pesquisa envolve altos custos e riscos e todos dividem os ônus e bônus da inovação. O maior desafio neste caso é influenciar a direção da inovação no sentido de obter contribuições que serão rentáveis para a empresa.

$\mathrm{Na}$ medida em que as incertezas típicas da fase de pesquisa vão sendo contornadas, forma-se uma constelação de valor por meio do adensamento dos participantes da rede, dentro da qual se realiza uma troca complexa de conhecimentos e tecnologias visando a consolidação de um padrão tecnológico. Este modo é recomendado quando a empresa detém os conhecimentos-chave e quer manter a integridade de um sistema, usando a rede para acelerar o desenvolvimento de inovações incrementais. O maior desafio é entender a necessidade dos clientes e ter a capacidade de desenhar sistemas que desmembrem os problemas maiores em partes discretas, nas quais cada parceiro possa trabalhar de forma relativamente autônoma. 
Já nos corretores virtuais qualquer empresa ou parceiro pode propor problemas ou oferecer soluções específicas e com contorno bem definido, e cada um decide que solução adotar. Este modo é recomendado nos casos de tecnologias maduras, com uma arquitetura modular consolidada e quando a empresa pode usar os conhecimentos gerados na rede para melhorar seus próprios produtos e processos. O maior desafio é criar valor com o conhecimento trocado na rede em proporção maior do que o valor que seria criado mantendo a inovação dentro da empresa. Este tipo de comunidade também pode funcionar para aproximar compradores e vendedores de propriedade intelectual. Nestas redes, os relacionamentos são altamente flexíveis, sendo vital que sejam mantidos mecanismos de incentivo que fortaleçam o adensamento contínuo da comunidade vis-à-vis as comunidades com tecnologias concorrentes. Detalham a seguir os determinantes do grau de virtualidade da rede de colaboração.

No arcabouço proposto, o grau de virtualidade das REVs depende da ação dos direcionadores da virtualidade, os quais agem sobre o conjunto de características que definem os tipos de REVs. Como já foi visto em 2.6, a virtualidade no ambiente empresarial está primariamente relacionada ao desenvolvimento de unidades operacionais interligadas logicamente por meio de intensas comunicações mediadas por computadores (Chandrashekar e Schary, 1999; Markus et al., 2000). De acordo com os resultados da revisão da literatura, podem-se considerar pelo menos cinco atributos principais da virtualidade: i) independência de tempo e espaço para a execução dos processos de negócio; ii) ruptura entre oferta de soluções e a propriedade dos meios físicos para desenvolvê-las; iii) metaestratégia, ou seja, a estratégia que emerge ao nível da rede de relacionamentos e que não estava ao alcance de cada uma das empresas isoladamente; iv) autogestão; v) geometria variável (Castells, 1999).

Em outros termos, uma rede de colaboração pode ser classificada como "virtual" quando: i) as barreiras geográficas e temporais são irrelevantes para estabelecer parcerias; ii) a rede é capaz de acompanhar rapidamente os requerimentos dos clientes, usando o acesso aos recursos oferecidos por quaisquer parceiros para a inovar, os quais tomam por base, para oferecer seus componentes, a configuração técnica predefinida para cada solução; iii) o potencial de atualização, nos limites das inovações incrementais, é maximizado pela integração dos sistemas de cada parceiro e pelo uso de agentes inteligentes e de padrões lógicos de interface; iv) os encontros são pontuais, estabelecidos apenas para atender ad hoc a uma configuração de recursos. Cada solução, que é um componente da arquitetura modular, requererá uma configuração e, por conseguinte, um conjunto de parceiros; v) a rede se autogerencia tomando por base os mecanismos de coordenação e padrões de conexão previamente estabelecidos, apoiando-se nas métricas de apuração da contribuição e de avaliação de confiança disponibilizadas na rede para a solução de conflitos. De acordo com o modelo proposto, estes atributos da virtualidade influenciariam e seriam influenciados pelos atributos da OI, descritos na tabela 2. As influências dos direcionadores da virtualização sobre os atributos da virtualidade são discutidas a seguir.

A natureza lógica — ou virtual — das conexões, em contraposição às características físicas ou interpessoais que predominam nos relacionamentos tradicionais, possibilita o acesso a um capital informacional novo. Isto se deve ao uso intenso das TICs interligando os processos 
de negócio na rede, o que, por sua vez, permite a troca de informações em tempo real, geradas do compartilhamento de bases de dados e aplicativos, os quais se apoiam em regras de negócio predefinidas para tomar decisões limitadas a seu escopo de atuação. Mesmo nos casos em que os sistemas não consigam "tomar decisões", é possível fazê-los disparar alertas eletrônicos, dentro de um workflow predefinido, sempre que um determinado parâmetro desviar-se de uma meta ou de um comportamento esperado. Assim, a possibilidade de construírem-se laços lógicos, de natureza colaborativa, e de explorá-los em conjunto com os parceiros de negócio, abre novas possibilidades para a construção de conhecimento explícito, o que condiciona o grau de virtualização das REVs na medida em que enriquece o processo de formulação de metaestratégias.

O grau de modularidade é um importante direcionador na medida em que condiciona a virtualização das REVs, entre outros aspectos, ao permitir que a empresa não tenha dificuldades para encontrar, independentemente dos limites de tempo e espaço, os parceiros para o suprimento dos componentes da solução oferecida pela rede. Ademais, ele torna a implementação de inovações incrementais mais efetiva, ao permitir que elas possam ser introduzidas em cada componente, sem a necessária alteração da arquitetura do produto. Assim, a atualização que a virtualização permite tornará a gestação de uma metaestratégia qualitativamente diferente daquela gerada nos limites permitidos por uma arquitetura monolítica. Cadeias de valor outrora verticalizadas, uma vez modularizadas, podem ser mais facilmente desmembradas e transferidas para parceiros independentes, facilitando a descontinuidade entre soluções e meios físicos.

A cultura virtual inspirada nos valores adotados na gestão das linguagens em código aberto é um direcionador da virtualidade crítico porquanto influencia a predisposição dos executivos de: i) criarem conjuntamente o valor com seus parceiros; ii) abrirem mão de ganhos imediatos em troca da formulação no longo prazo de soluções inovadoras; iii) submeterem-se às regras coletivas de conduta; iv) adotarem métricas claras, amplamente disponíveis, que meçam a credibilidade de cada parceiro. A adoção destes valores terá forte influência na capacidade que a REV terá de se autogerir em acordo com as regras de convivência esperadas em uma sociedade em redes (Castells, 1999). Na medida em que impacta também na predisposição que os executivos terão em compartilharem informações estratégicas e integrarem mais efetivamente os processos de negócios com seus parceiros, uma cultura virtual, aqui associada aos modelos de open source, potencializa os resultados de uma metaestratégia.

Uma infraestrutura de TI, que envolva o compartilhamento de bases de dados, é um requisito para a geração de soluções formuladas pela configuração dinâmica de forças e restrições tratadas por agentes inteligentes, os quais interpretam os movimentos de mercado a partir de regras de negócio cada vez mais sofisticadas. A intensa mediação de computadores na automação de processos de negócio e na troca de informação para a execução das atividades empresariais foi a precondição para a criação da independência de tempo e espaço na gestão dos negócios e para permitir a descontinuidade entre a solução de uma necessidade e a propriedade dos meios físicos para desenvolvê-la. No caso do processo inovativo, esta infra- 
estrutura é a base para a adoção de tecnologias para prototipação e simulação, que ampliam a virtualização do processo inovativo.

Um hub integrador, que ofereça uma infraestrutura de TI independente, parece ser um requisito para a condução para a criação e compartilhamento de conhecimento nos movimentos de código aberto. Logo, o uso de portais de internet, também chamados de infomediaries, permite a participação dos parceiros em condições de garantia da máxima independência de tempo e espaço, já que as empresas podem se encontrar a qualquer momento e a partir de qualquer lugar, bastando que tomem por base as regras de comportamento estabelecidas pela comunidade. Pela dinâmica dos relacionamentos, a geometria destas REVs é variável em acordo com cada solução de acoplamento. Dada a absoluta observância dos mecanismos de coordenação preestabelecidos, a rede é capaz de se autorregular.

Nos estudos de casos a respeito do uso de OI na gestão de P\&D conduzidos por Hacievliyagil (2007), há evidências de que as firmas intensificam seus esforços de codificação de conhecimentos e engajaram-se na implantação de TICs para suportar a troca das informações nos fluxos de conhecimento com agentes externos. Indo além, o papel da virtualidade habilitada pelas TICs na mudança em direção ao modelo de OI é ressaltado na literatura (Dogson et al., 2006; Pisano e Verganti, 2008). Verona e colaboradores (2006), por seu turno, destacam o papel de corretores virtuais de conhecimento (virtual knowledge brokers) na intermediação das necessidades de inovação dos clientes e a rede de pesquisadores externos a eles associados. Nesta pesquisa, este uso será analisado a partir dos recursos aportados na rede pela empresa que está no centro da análise.

\section{Observações finais}

O objetivo deste artigo era articular as bases conceituais de um arcabouço analítico voltado para estudo da virtualidade habilitada pelas TICs nas estratégias de inovação aberta. Entendese que o resultado pretendido foi alcançado na medida em que o artigo apresenta, tendo por base uma ampla e profunda revisão da literatura científica pertinente a este objetivo, a proposta de articulação conceitual, descrevendo seus argumentos centrais e analisando, dentro dos limites impostos por este artigo, o funcionamento da sua estrutura analítica. Como primeira evidência, este estudo sugere que os gestores de P\&D das empresas brasileiras podem se valer dos atributos da OI para, nas condições de contorno adequadas, acelerar o processo de aprendizagem tecnológica, contornando em parte as limitações típicas dos SNI de países emergentes, sem, no entanto, se descuidarem de desenvolver e manter a capacidade de absorver os conhecimentos disponíveis além de suas fronteiras.

No que diz respeito ao potencial de contribuição do ensaio para o campo de gestão da inovação, o pesquisador entende que os resultados ajudam a teoria mostrando que as estratégias de inovação aberta podem ser potencializadas pelo uso da virtualidade em acordo com os objetivos pretendidos em cada tipo de REVs. Entende-se que o estudo do uso virtualidade no processo de inovação tem um grande potencial para pesquisas futuras no campo de estra- 
tégias de inovação aberta nas firmas brasileiras. Cumpre ressaltar, no entanto, que o modelo aqui oferecido, ainda que esteja apoiado em arcabouços validados cientificamente, carece de aplicação empírica, o que limita qualquer conclusão tirada a partir dele.

Do ponto de vista da prática gerencial na área de $\mathrm{P} \& \mathrm{D}$, os resultados mostram que, com a especialização e a dispersão geográfica crescente da produção de conhecimento, argumento central da OI, a construção de uma arquitetura de TI que permita a troca fluida de conhecimentos em escala global assume relevância ainda maior nas estratégias de busca de conhecimentos externos. Além disto, os gestores de P\&D das firmas brasileiras devem avaliar com especial interesse a pertinência de se adquirir TICs, tais como ferramentas de simulação e prototipação, que possam apoiar seus pesquisadores na virtualização do processo inovativo. Ressalta-se que, em indústrias de base tecnológica, tal como biotecnologia, a ausência destas TICs - e de especialistas aptos a operá-las — pode se converter em um sério limitador do processo inovativo e, por conseguinte, da capacidade de competir em escala global. As evidências coletadas sugerem que, nestas indústrias, as TICs aplicadas na P\&D converteram-se em elementos críticos da capacidade tecnológica para o desenvolvimento de novos produtos e dos processos produtivos correlatos. Por fim, a virtualização na cadeia de P\&D pode se transformar num elemento importante na capacidade de a firma brasileira integrar-se em cadeias globais de produção de conhecimento, acelerando o processo de aprendizagem na direção de níveis mais avançados de capacidades tecnológicas inovadoras.

\section{Referências}

ACHROL, Ravi S.; KOTLER, Philip. Marketing in the network economy. Journal of Marketing, v. 63 (special issue), p. 146-163, 1999.

ALAVI, M.; LEIDNER, D.E. Knowledge management and knowledge management systems: conceptual foundation and research issues. MIS Quarterly, v. 1, n. 25, p. 107-136, 2001.

ALBERTIN, L.A. Valor estratégico dos projetos de tecnologia da informação. Revista de Administração de Empresas - RAE, v. 41, n. 3, p. 42-50, 2001.

AUDRETSCH, D.; FELDMAN, M.P. R\&D spillovers and the geography of innovation and production. American Economic Review, v. 86, n. 3, p. 630-640, 1996.

BELL, M.; PAVITT, K. The development of technological capabilities. In: HAQUE, I.U. (Ed.). Trade, technology and international competitiveness. Washington, DC: World Bank, 1995.

BLACK, J.A.; EDWARDS, S. Emergence of virtual or network organizations: fad or feature. Journal of Organization Change, v. 13, n. 6, p. 567-576, 2000.

BORGHOFF, U.; PARESCHI, R. Information technology for knowledge management. Berlin: SpringerVerlag, 1998. 
BROWN, J.S. Foreword: innovating innovation. In: CHESBROUGH, H. Open innovation. Cambridge: Harvard Business Scholl Press, 2003.

CAPRA, F. As conexões ocultas: ciência para uma vida sustentável. São Paulo: Cultrix, 2002.

CASTELLS, M. A sociedade em rede. São Paulo: Paz e Terra, 1999.

CHANDLER, A. The visible hand. Cambridge: Harvard School Business Press, 1977.

CHANDRASHEKAR, A.; SCHARY, P.B. Toward the virtual supply chain: the convergence of IT and organization. The International Journal of Logistics Management, v. 10, n. 2, 1999.

CHESBROUGH, H.W. Open innovation: a new paradigm for understanding industrial innovation. In: CHESBROUGH, H.W.; VANHAVERBEKE, W.J. Open innovation: researching a new paradigm. New York: Oxford University Press, 2006.

CHESBROUGH, H.W. Open innovation: the new imperative for creating and profiting from technology. Cambridge: Harvard Business Scholl Press, 2003.

CHESBROUGH, H.W.; APPLEYARD, M.M. Open innovation and strategy. California Management Review, v. 50, n. 1, p. 57-76, 2007.

CHESBROUGH, H.W.; CROWTHER, A.K. Beyond high tech: early adopters of open innovation in other industries. R\&D Management, v. 36, n. 3, p. 229-236, 2006.

CHESBROUGH, H.W.; TEECE, D.J. When is virtual virtuous? Harvard Business Review, v. 74, n. 1, p. 65-73, 1996.

COHEN, W.M.; LEVINTHAL, D.A. Absorptive capacity: a new perspective on learning and innovation. Administrative Science Quarterly, v. 35, p. 128-152, 1990.

COOMBS, R.; METCALFE, J.S. Organizing for innovation: co-ordinating distributed capabilities. In: FOSS, N.; MAHNKE, V. Competence, governance and entrepreneurship. London: Oxford University Press, 2000.

DAHLANDER, L.; GANN, D.M. How open is innovation? Research Policy, v. 39, p. 699-709, 2010.

DANTAS, E.; BELL, M. Latecomer firm and the emergence and development of knowledge networks: the case of Petrobras in Brazil. Research Policy, v. 38, p. 829-844, 2009.

DOGSON, M.; GANN, D.; SALTER, A. The role of technology in the shift towards open innovation: the case of Procter \& Gamble. R\&D Management, v. 36, n. 3, p. 333-346, 2006.

DOSI, G. Technological paradigms and technological trajectories. Research Policy, v. 11, n. 3, p. 147-162, 1982.

DUTRÉNIT, G. El papel de las rutinas en la codificación del conocimiento en la firma. Revista Análisis Econômico, v. 34, n. 16, p. 211-230, 2001.

DYER, J.H.; SINGH, H. The relational view: cooperative strategy and sources of interorganizational competitive advantage. Academy of Management Review, v. 23, n. 4, p. 660-679, 1998. 
FAUCHEUX, C. How virtual organizing is transforming management science. Communications of the ACM, v. 40, n. 9, p. 50-56, 1997.

FIGUEIREDO, P.N. Gestão da inovação: conceitos, métricas e experiências de empresas no Brasil. Rio de Janeiro: LTC, 2009.

FREEMAN, C.; SOETE, L. The economics of industrial innovation. 3. ed. London: Frances Pinter, 1997.

GHOSH, M.; JOHN, G. Governance value analysis and marketing strategies. Journal of Marketing, v. 63, p. 131-143, special issue, 1999.

GRANT, R.M. Toward a knowledge-based theory of the firm. Strategic Management Journal, v. 17, p. 109-122, Winter special issue, 1996.

GULATI, R. Alliances and networks. Strategic Management Journal, v. 19, p. 293-317, 1998.

GULATI, R.; NOHRIA, N.; ZAHEER, A. Strategic networks. Strategic Management Journal, v. 21, p. 203-215, 2000.

HACIEVLIYAGIL, N.K. The impact of open innovation on technology transfers at Philips and DSM. Thesis (M.Sc.) — Faculty of Technology, Policy \& Management, Delft University of Technology, 2007.

HÄCKI, R.; LIGHTON, J. The future of the networked company. The McKinsey Quarterly, n. 3, p. 26-39, 2001.

HANNAN, M.; FREEMAN, J. The population ecology of organizations. American Journal of Sociology, V. 82, p. 929-964, 1977.

HUGHES, B.; WAREHAM, J. Knowledge arbitrage in global pharma: a synthetic view of absorptive capacity and open innovation. R\&D Management, v. 40, n. 3, p. 324-343, 2010.

HUSTON, L.; SAKKAB, N. Conectar e desenvolver: como funciona o novo modelo de inovação da Procter \& Gamble. Harvard Business Review, 2006.

JARRILLO, J.C. On strategic networks. Strategic Management Journal, v. 9, p. 31-41, 1998.

KEEN, P.G.W. Shaping the future: business design trough information technology. Cambridge: Harvard Business School Press, 1991.

KIM, L. Crisis construction and organizational learning: capability building in catching-up at Hyundai Motor. Research Policy, v. 9, p. 506-521, 1998.

KOGUT, B.; ZANDER, U. Knowledge of the firm, combinative capabilities, and the replication of technology. Organization Science, v. 3, p. 383-397, 1992.

LANDES, S.D. Prometeu desacorrentado: transformação tecnológica e desenvolvimento industrial na Europa Ocidental desde 1750 até nossa época. Rio de Janeiro: Nova Fronteira, 1994. [1. ed.: 1969]

LAURSEN, K.; SALTER, A. Open for innovation: the role of openness in explaining innovation performance among U.K. manufacturing firms. Strategic Management Journal, v. 27, p. 131-150, 2006. 
LEE, K.; LIM, C. Technological regimes, catching-up and leapfrogging: findings from the Korean industries. Research Policy, v. 30, p. 459-483, 2001.

LEVITT, B.; MARCH, J.G. Organizational learning. Annual Review of Sociology, v. 14, p. 319-340, 1988.

LÉVY, P. Qué es lo Virtual? Barcelona: Paidós, 1999.

LUNDVALL, B.A. National systems of innovation: towards a theory of innovation and interactive learning. London: Pinter Publishers, 1992.

MACEDO-SOARES, T.D.L.V.A. Strategic alliances and networks: conceptual tools for strategic assessments. Proceedings of GBATA Conference, Rome, 2002.

MALERBA, F. Sectoral systems of innovation and production. Research Policy, v. 31, p. 247-264, 2002.

MARKUS, M.L.; MANVILLE, B.; AGRES, C.E. What makes a virtual organization work? Sloan Management Review, v. 42, n. 1, p. 13, 2000.

MOWSHOWITZ, A. Virtual organization. Communications of the ACM, v. 40, n. 9, p. 30-37, 1997.

NAMBISAN, S. Designing virtual customer environments for new product development: toward a theory. Academy of Management Review, v. 27, n. 3, p. 392-413, 2002.

NAMBISAN, S.; SAWHNEY, M. A buyer's guide to the innovation bazaar. Harvard Business Review, p. 109-118, June 2007.

NELSON, R.R.; WINTER, S.G. An evolutionary theory of economic change. Cambridge: Harvard University Press, 1982.

NONAKA, I. A dynamic theory of organizational knowledge creation. Organization Science, v. 5, n. 1, p. 14-37, 1994.

NONAKA, I.; TAKEUCHI, H. The knowledge creating firm: how Japanese firms create the dynamics of innovation. New York: Oxford University Press, 1995.

ORSENIGO, L.; PAMMOLLI, F.; RICCABONNI, M. Technological change and network dynamics: lessons from the pharmaceutical industry. Research Policy, v. 30, p. 485-508, 2001.

PAVITT, K. Sectoral patterns of technical change: towards a taxonomy and a theory. Research Policy, v. 13, p. 343-373, 1984.

PAVITT, K. What we know about the strategic management of technology. California Management Review, p. 17-96, 1990.

PENROSE, E. The theory of the growth of the firm. New York: Oxford University Press, 1959.

PISANO, G.P. Profiting from innovation and the intellectual property revolution. Research Policy, n. 35, p. 1122-1130, 2006.

PISANO, G.P.; VERGANTI, R. Which kind of collaboration is right for you? Harvard Business Review, v. 86, n. 12, p. 78-86, 2008. 
PITASSI, C. O papel de um centro de P\&D em empresas de ramos tradicionais: o caso da Unidade de Logística da Vale. Cadernos EBAPE, 2011 (no prelo).

PITASSI, C. Redes estratégicas virtuais: uma pesquisa exploratória. Tese (doutorado) — Instituto de Administração e Gerência, Pontifícia Universidade Católica do Rio de Janeiro, Rio de Janeiro, 2004.

PITASSI, C.; DIAS, R.M. Redes estratégicas virtuais: um estudo de caso do portal Covisint GM-FIAT WWP. In: CONGRESSO DA ANPAD, 27ํㅡ, Atibaia, 2003. Anais...

PITASSI, C.; MACEDO-SOARES, T.D.L.v.A. Redes estratégicas virtuais: fatores críticos de sucesso. Revista de Administração Contemporânea — RAC, edição especial, v. 7, p. 75-99, 2003.

POLANYI, M. The tacit dimension. New York: Anchor Day, 1966.

ROBERTSON, P.L.; VERONA, G. Post-Chandlerian firms: technological change and firm boundaries. Australian Economic History Review, v. 46, n. 1, p. 70-94, 2006.

ROSENBERG, N. Inside the black box: technology and economics. Cambridge: Cambridge University Press, 1982.

ROTHWELL, R. Industrial innovation: success, strategy, trends. In: DODGSON, M.; ROTHWELL, R. (Ed.). The handbook of industrial innovation. Hants: Edward Elgar, 1994.

SAKKAB, N. Connect \& develop complements research \& develop at P\&G. Research-Technology Management, v. 45, p. 38-45, 2002.

SANCHEZ, R. Modular architectures in the marketing process. Journal of Marketing, v. 63, p. 92111, special issue, 1999.

SCHUMPETER, J. The theory of economic development. Cambridge: Harvard UP, 1934.

SHAPIRO, C.; VARIAN, H.R. Information rules: a strategic guide to the network economy. Boston: Harvard Business School Press, 1999.

SIVADAS, E.; DWYER, R.F. An examination of organizational factors influencing new product success in internal and alliance-based processes. Journal of Marketing, v. 64, p. 31-49, 2000.

TEECE, D. Profiting from technological innovation: implications for integration, collaboration, licensing and public policy. Research Policy, v. 15, n. 6, p. 285-305, 1986.

TEECE, D.; PISANO, G.; SHUEN. Dynamic capabilities and strategic management. Strategic Management Journal, v. 18, n. 7, p. 509-533, 1997.

THOMKE, S. Experimentation matters. Boston: Harvard Business Scholl Press, 2003.

TROTT, P.; HARTMANN, D. Why “open innovation" is old wine in new bottles. International Journal of Innovation Management, v. 13, n. 4, p. 715-736, 2009.

TURBAN, E.; RAINER JR., R.K.; POTTER, R.E. Introduction to information systems. New York: John Wiley \& Sons, Inc, 2007. 
UTTERBACK, J.M. The process of technological innovation within the firm. Academy of Management Journal, v. 14, n. 1, p. 75-88, 1971.

UZZI, B. Social structure and competition in interfirm networks: the paradox of embeddedness. Administrative Science Quarterly, v. 42, p. 36-67, 1997.

VENKATRAMAN, N.; HENDERSON, J.C. Real strategies for virtual organizing. Sloan Management Review, v. 40, n. 1, p. 33-48, 1998.

VERONA, G.; PRANDELLI, E.; SAWHNEY, M. Innovation and virtual environment: toward virtual knowledge brokers. Organization Studies, v. 27, n. 6, p. 765-788, 2006.

VON HIPPEL, E. Shifting innovation to users via toolkits. Management Science, v. 48, n. 7, p. 821833, 2002.

ZUBOFF, S. In the age of the smart machine. New York: Basic Books, 1988.

Claudio Pitassi é professor adjunto do Mestrado de Administração de Empresas (Made) da Universidade Estácio de Sá (Unesa). E-mail: claudio.pitassi@gmail.com. 